\title{
Hepatite aguda secundária ao abuso de cocaína, associada a severa rabdomiólise
}

\author{
Acute hepatitis secondary to abuse of cocaine, associated with severe rhabdomyolysis
}

\section{José Hícaro Hellano Gonçalves Lima Paiva ${ }^{1}$, Vitória Nunes Medeiros ${ }^{2}$, Ivelise Regina Canito Brasil ${ }^{3}$}

Paiva JHHGL, Medeiros VN, Brasil IRC. Hepatite aguda secundária ao abuso de cocaína, associada a severa rabdomiólise / Acute hepatitis secondary to abuse of cocaine, associated with severe rhabdomyolysis. Rev Med (São Paulo). 2020 mar.-abr.;99(2):189-96.

RESUMO: A cocaína é uma droga com notória capacidade de afetar adversamente quase todos os órgãos do corpo e pode causar uma infinidade de anormalidades multisistêmicas secundárias. No presente estudo, relatamos três casos de insuficiência hepática aguda complicada por rabdomiólise e lesão renal aguda após consumo de cocaína e álcool. O início da doença, as manifestações clínicas, os dados laboratoriais, o diagnóstico e a terapêutica de cada paciente foram registrados. Nota-se a presença de causas multifatoriais para a ocorrência de Insuficiência Hepática e Rabdomiólise nos três casos relatados. Os testes laboratoriais revelaram que a concentração sérica de transaminases, bem como os de creatinafosfoquinase (CPK) aumentaram e que os sintomas de insuficiência renal aguda estavam presentes, o que forneceu um diagnóstico exato de insuficiência hepática aguda complicada por grave rabdomiólise e lesão renal aguda dialítica. Rabdomiólise é uma condição de hipermioglobinúria, sendo uma dos principais fatores que fomentam uma piora da função renal. A terapia da fase inicial envolveu suporte a ressuscitação volêmica associada a terapia dialítica com rigoroso controle da função renal. Os três pacientes desenvolveram comprometimento de múltiplos órgãos, mas apenas um foi a óbito devido a gravidade do quadro. Considerando os três casos apresentados, concluímos que a insuficiência hepática, complicada por rabdomiólise, secundária ao uso de cocaína e álcool pode ter mau prognóstico clínico, a depender de vários fatores. $\mathrm{E}$ os médicos devem estar cientes dos potenciais efeitos causados pela cocaína, a fim de gerir as múltiplas complicações associadas ao abuso destas substâncias.

Descritores: Cocaína; Rabdomiólise; Lesão renal aguda; Doença hepática induzida por substâncias e drogas.

\begin{abstract}
Cocaine is a drug with a noticeable ability to adversely affect almost every organ in the body and can cause a multitude of secondary multisystem abnormalities. In the present study, we report three cases of acute liver failure complicated by rhabdomyolysis and acute kidney injury following cocaine and alcohol use. The disease onset, clinical manifestations, laboratory data, diagnosis and treatment of each patient were recorded. Note the presence of multifactorial causes for the occurrence of liver failure and rhabdomyolysis in the three reported cases. Laboratory tests revealed that serum trasaminases as well as creatine phosphokinase (CPK) concentrations increased, and symptoms of acute renal failure were present, which provided an accurate diagnosis of acute liver failure complicated by severe rhabdomyolysis and acute renal injury. dialytic rhabdomyolysis is a condition of hypermyoglobinuria, being one of the main factors that promote a deterioration of renal function. Early phase therapy involved support for volume resuscitation associated with dialysis therapy with strict control of renal function. All three patients developed multiple organ involvement, but only one died due to the severity of the condition. Considering the three cases presented, we conclude that liver failure, complicated by rhabdomyolysis, secondary to cocaine and alcohol use may have a poor clinical prognosis, depending on several factors. And doctors should be aware of the potential effects caused by cocaine in order to manage the multiple complications associated with substance abuse.
\end{abstract}

Keywords: Cocaine; Rhabdomyolysis; Acute kidney injury; Chemical and drug induced liver injury.

1. Faculdade de Medicina da Universidade Estadual do Ceará (UECE), Fortaleza, Ceará, Brasil. ORCID: https://orcid.org/0000-0002-6232-003X. E-mail: hellanohicaro@gmail.com.

2. Faculdade de Medicina da Universidade Estadual do Ceará (UECE), Fortaleza, Ceará, Brasil. ORCID: https://orcid.org/0000-0003-1621-2385. E-mail: vitoria21nunes@gmail.com.

3. Serviço de Transplante do Hospital Geral de Fortaleza (HGF), Fortaleza, Ceará, Brasil. ORCID: https://orcid.org/0000-0002-4886-7442. E-mail: ivelise.canito@uece.br.

Endereço para correspondência: José Hícaro Hellano Gonçalves Lima Paiva. Rua Pão de Açúcar, 114 - Centro - Reriutaba. e-mail: hellanohicaro@ gmail.com 


\section{INTRODUÇÃO}

Tntoxicação e abuso de cocaína configuram-se 1 como uma problema global, que estão associados a diversas complicações, resultando em elevada morbimortalidade dos usuários ${ }^{1}$. Apesar da queda de usuários de cocaína entre 2007 e 2014, a prevalência de usuários é significativa, com cerca de 18,3 milhões de usuários em todo o mundo. Nesse contexto estima-se que a prevalência global de doenças atribuíveis ao uso de álcool e drogas ilícitas seja cerca de $5,4 \%{ }^{2}$.

No Brasil, o consumo de cocaína é uma condição prevalente, sendo um dos principais países resposáveis pelo comérico ilegal da droga. Vale destacar que o uso da substância se mostra mais comum na faixa etária de 18 -24 anos $^{2}$. Há um aumento significativo no consumo de cocaína, apesar de uma redução no consumo nos Estados Unidos e relativa estabilidade na Europa. A crescente comercialização dessa substância em áreas de fragilidade socioeconômica e que têm pouco acesso a um sistema de reabilitação é uma das justificativas para esse aumento ${ }^{3}$.

A síndrome clínica dos paciente admitidos em setores de emergências por intoxicação de cocaína é caracterizada por manifestações simpatomiméticas (taquicardia, hipertensão, agitação, taquipneia e diaforese) e/ou por manifestações sistêmicas ${ }^{1}$.

As alterações neurológicas agudas podem se manifestar com flutuações do nível de consciência, mania, psicose, convulsões generalizadas e acidentes vasculares cerebrais. As manifestações cardiovasculares podem variar desde uma queixa de dor torácica aguda, a qual é a mais frequente, até dissecção aguda da aorta ${ }^{4}$ e arritmias supraventriculares ou ventriculares ${ }^{1}$ (Figura 1).

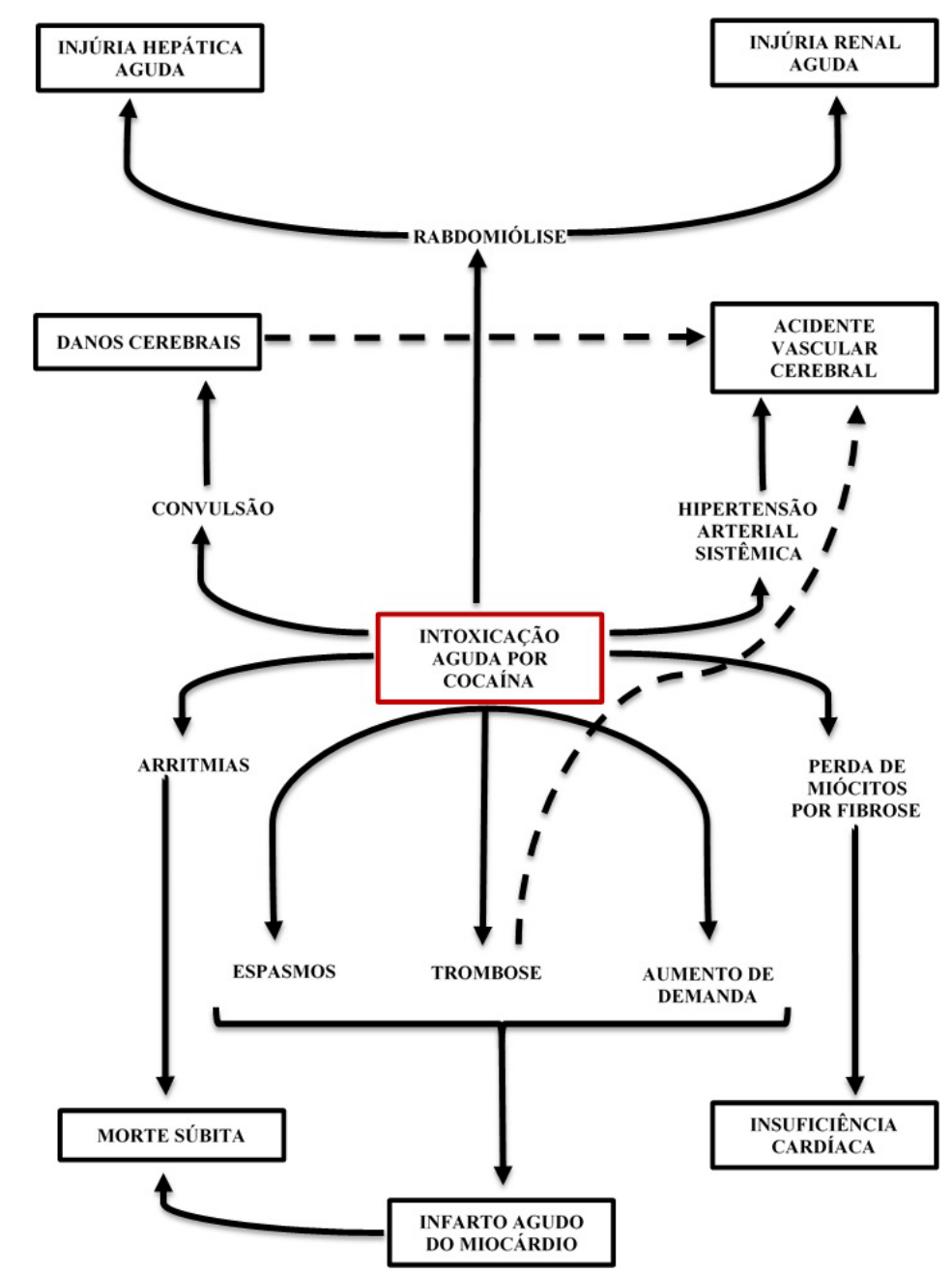

Fonte: Elaborado pelos autores.

Figura 1. Complicações associadas ao uso de cocaína e possíveis mecanismos

Tais condições podem inferir acerca do potencial de morbi-mortalidade associados ao abuso de cocaína. Apesar de bem documentados os efeitos que a cocaína causa nos sistemas supracitados, pouco se sabe sobre os efeitos e manejo de pacientes com acometimento renal e hepático ${ }^{5}$. A hepatotoxicidade é uma complicação que pode 
ocorrer após uso de copiosa quantidade de cocaína. A insuficiência hepática aguda é definida a partir de alterações dos parâmetros de coagulação e dos status mental (encefalopatia) em pacientes sem cirrose pré-existente, e que apresentem doença com menos 26 semanas de duração $0^{6,7}$.

A patogênese da hepatite aguda grave secundária a intoxicação por cocaína ainda é incerta. No entanto, acredita-se que a metabolização da cocaína resulta no acúmulo de radicais livres no sistema hepático, causando estresse oxidativo dos hepatócitos. Além disso, existem outras condições que podem agir em sinergismos à agressão hepática, como abuso de álccol, rabdomiólise e o uso de outras substâncias hepatotóxicas ${ }^{8,9}$.

Outra manifestação clínica importante é a rabdomiólise, a qual pode apresentar progressão clínica grave e, por vezes, fatal. Existem múltiplas causas associadas a essa condição como trauma, hipertermia, infecções, síndromes convulsivas e intoxicações exógenas ${ }^{10}$. Sua apresentação clínica é variável e pode se manifestar desde casos assintomáticas até casos graves, causando choque, síndrome compartimental, Injúria Renal Aguda, alterações hidroeletrolíticas e distúrbios acido-básicos ${ }^{11}$.

O diagnóstico laboratorial de rabdomiólise é feito a patir da dosagem de creatinofosfoquinasase sérica (CPK), a qual pode ser normal nas fases iniciais do quadro clínico. Entretanto, as alterações de função renal e hepática podem estar presentes desde o início dos sintomas ${ }^{12}$.

\section{OBJETIVOS}

O objetivo desse estudo consiste em descrever três casos de Hepatite Aguda Grave induzida por cocaína e complicada por Rabdomiólise e Injúria Renal Aguda, em pacientes adultos jovens.

\section{MÉTODOS}

Coleta de dados se deu por meio da análise de prontuário físico, no Hospital Geral de Fortaleza - cidade de Fortaleza, Ceará, Brasil. Foram tomados os cuidados para garantir o anonimato dos pacientes.

\section{RELATOS DE CASOS}

\section{Caso 1}

Paciente masculino, 32 anos, usuário de cocaína e alcoolista desde os 17 anos de idade, com uso regular quatro vezes na semana. Buscou assistência médica após uso de copiosa quantidade de cocaína, 3,5 gramas, e referia na admissão mialgia intensa, confusão mental de início recente e alterações urinárias, com urina escurecida e diminuição da diurese.
No exame admissional paciente apresentava-se ictérico, agitado, desorientado, escala de coma de glasgow de 11 , pressão arterial de 106x40 $\mathrm{mmHg}$, pulso de 56 batimentos por minuto, frequência respiratória com 18 incurssões respiratórias por minuto, saturação de oxigênio de $97 \%$ em ar ambiente, temperatura de $36.3^{\circ} \mathrm{C}$. O exame físico revelou dor abdominal difusa, demais sistemas não apresentavam alterações.

Os testes laboratoriais inicias revelaram aumento nos valores das enzimas hepáticas, dos marcadores de lesão renal, de leucócitos, de CPK e de Proteína-C Reativa (PCR). Outros parâmetros laboratorias avaliados encontravam-se dentro do valor de normalidade (Tabela 1).

Foram iniciados cuidados intensivos, bem como hidratação venosa vigorosa e monitoramento da diurese. No entanto, paciente evolui com piora da azotemia e anúria, sendo indicada hemodiálise.

Diante do quadro clínico, aventou-se a hipótese diagnóstica de Insuficiência Hepática Aguda secundária ao uso de cocaína, de acordo com os critérios da The American Association for the Study of Liver Diseases. Além disso, apresentou Insuficiência Renal Aguda oligúrica, de acordo com critérios da Kidney Disease Improving Global Outcomes (KDIGO), secundária a grave rabdomiólise.

Durante internamento hospitalar, paciente persistiu com desorientação temporo-espacial, evoluindo ainda com sangramento ativo ocular e em óstio de acesso venoso central. Apresentou também picos febris com temperaturas de $38^{\circ} \mathrm{C}$ e leucocitose com predomínio de polimorfonucleares, sendo aventada a hipótese de infecção bacteriana superajuntada ao quadro clínico. Foi iniciada antibioticoterapia com Metranidazol e Ciprofloxacino em virtude da suspeita clínica de colangite.

A despeito dos cuidados intensivos, no seguimento paciente evoluiu com hipoglicemia refratária a medida farmacológicas, elevação persistente de enzimas hepáticas e bilirrubinas, além de plaquetopenia e anemia severa. Transfusão de quatro concentrados de hemácias foi necessária, com melhora subsequente dos parâmetros hematimétricos.

No quinto dia de internamento hospitalar paciente apresentou piora dos parâmetros laboratoriais e suspeita de síndrome compartimental. Foi realizada avaliação ultrassonográfica de membro superior esquerdo, a qual revelou presença de duas coleções sanguinolentas. Diante disso, realizou-se terapia transfusional com um concentrado de hemácias, e antibióticos foram escalonado para Meropenem e Vancomicina, associando-se terapia antifúngica com anidulafugina.

Sorologias para hepatites virais e lepstospirose, fator $\mathrm{V}$ de Leiden, urocultura e hemocultura foram negativas. Os exames de imagem realizados - Tomografia Computadorizada de crânio, tórax e abdômen - não revelaram alterações importantes. 
Paiva JHHGL, et al. Hepatite aguda secundária ao abuso de cocaína, associada a severa rabdomiólise.

No dias subsequentes ao internamento, paciente evolui com melhora progressiva dos parâmetros clínicos e laboratoriais, sendo optado por conduta expectante quanto a necessidade de transplante hepático, uma vez que os critérios de King's College não foram preenchidos. Após 29 dias de internamento hospitalar paciente recebe alta para prosseguir com seguimento ambulatorial.

Tabela 1. Exames laboratoriais admissionais e durante o internamento hospitalar dos pacientes

\begin{tabular}{|c|c|c|c|c|c|c|c|c|c|c|c|c|}
\hline & \multicolumn{4}{|c|}{ Paciente 1} & \multicolumn{3}{|c|}{ Paciente 2} & \multicolumn{4}{|c|}{ Paciente 3} & \multirow[t]{2}{*}{ Ref. } \\
\hline Parâmetros & $1^{\text {a }}$ DIH & $2^{\mathrm{a}} \mathrm{DIH}$ & $5^{\text {a DIH }}$ & $10^{\mathrm{a}}$ DIH & $1^{\mathrm{a}} \mathrm{DIH}$ & $2^{\mathrm{a}}$ DIH & $3^{\text {a DIH }}$ & $1^{\text {a }}$ DIH & $2^{\mathrm{a}}$ DIH & $\begin{array}{c}5^{\text {a }} \\
\text { DIH }\end{array}$ & $\begin{array}{l}10^{\mathrm{a}} \\
\text { DIH }\end{array}$ & \\
\hline Potássio (mmol/l) & 3.4 & 2.8 & 5 & 3.8 & 4.3 & $* *$ & 4.9 & 3.7 & 5.7 & 5.3 & 4.6 & $3.5-5.3$ \\
\hline Sódio (mmol/1) & 141 & 133 & 132 & 131 & 138 & 137 & 135 & 137 & 140 & 138 & 136 & $\begin{array}{l}135- \\
148\end{array}$ \\
\hline BT (mmol/l) & 2.4 & 3.7 & 12.08 & 17.47 & $* *$ & 3.7 & 4.7 & 5.72 & 11.37 & 15.66 & 11.06 & \\
\hline $\mathrm{BD}(\mathrm{mmol} / \mathrm{l})$ & 0.7 & 1.5 & 7.24 & 12.2 & $* *$ & 3.2 & 3.6 & 3.53 & 5.05 & 6.75 & 8.61 & $<1$ \\
\hline Ureia (mg/dl) & 58 & 82 & 62 & 324 & 81 & 100 & 64 & 147 & 134 & 111 & 175 & $13-43$ \\
\hline Creatinina (mg/dl) & 2.9 & 5.3 & 5.2 & 6.7 & 5.3 & 6.9 & 5.5 & 4.57 & 7.4 & 6 & 7.7 & $0.6-1.1$ \\
\hline $\operatorname{AST}(\mathrm{U} / \mathrm{L})$ & 171 & 1576 & 2163 & 370 & $* *$ & 3105 & 6179 & 6720 & 10020 & 3205 & 135 & $<32$ \\
\hline ALT (U/L) & 46 & 467 & 3307 & 411 & $* *$ & 2354 & 5120 & 3740 & 7226 & 4152 & 254 & $<31$ \\
\hline TAP (s) & 1.47 & 3.5 & 1.95 & 1.22 & 6 & 1.82 & 3 & 3.53 & 4.47 & 1.67 & 1.04 & $10-14$ \\
\hline TTPA (s) & $* *$ & $* *$ & 1.4 & 1.46 & 0.5 & $* *$ & 3.95 & 1.93 & 1.77 & 1.3 & 1.24 & $22-28$ \\
\hline Lipase (U/L) & 113.9 & 59.6 & $* *$ & $* *$ & $* *$ & $* *$ & $* *$ & $* *$ & 1495 & 192 & 65 & $13-60$ \\
\hline Amilase (U/L) & 115 & 246 & $* *$ & $* *$ & $* *$ & $* *$ & $* *$ & $* *$ & 616 & 118 & 58 & $<84$ \\
\hline LDH (U/L) & 821 & 3827 & $* *$ & $* *$ & $* *$ & $* *$ & $* *$ & $* *$ & $* *$ & 3784 & 1989 & $\begin{array}{l}230- \\
460\end{array}$ \\
\hline CPK (U/L) & 3860.6 & 1230 & 24388 & 9194 & 184328 & 86865 & $* *$ & 502000 & 263995 & 59190 & 14562 & $<170$ \\
\hline Hemoglobina (g/dl) & 14.4 & 14.2 & 5.5 & 8.4 & 13.8 & 11.6 & 10.9 & 14.7 & 12.2 & 11.6 & 10.4 & $11.3-5.2$ \\
\hline Leucócitos (/mm³) & 25410 & 24490 & 11100 & 17400 & 25190 & 15730 & 22780 & 21800 & 23900 & 14000 & 13300 & $\begin{array}{l}3600- \\
10000\end{array}$ \\
\hline Plaquetas $\left(/ \mathrm{mm}^{3}\right)$ & 196000 & 230000 & 47000 & 147000 & 310000 & 207000 & 306000 & 184000 & 123000 & 57000 & 398000 & $\begin{array}{l}150--50 \\
\left(10^{3}\right)\end{array}$ \\
\hline
\end{tabular}

Legenda: AST: Aspartato aminotransferase; ALT: Alanine aminotransferase; LDH: lactate desidrogenase; CPK: Creatinofososquinase; PT: tempo de protrombina; aPTT: Tempo de tromblopastina parcial ativada; Ref: Referência; DIH: Dia de internação hospitalar. ** Não avaliado

Fonte: Elaborado pelos autores

\section{Caso 2}

Paciente masculino, 33 anos, portador de dislipidemia e usuários crônico de cocaína. Foi admitido em Unidade de Terapia Intensiva após abuso de cocaína e politrauma, após quedas da própria altura secundária a surto psicótico.

No exame admissional apresentava-se com rebaixamento do nível de consciência, escala de coma de glasgow de 8 , pressão arterial sistêmica de $82 \times 41 \mathrm{mmHg}$, temperatura axilar de $36.2^{\circ} \mathrm{C}$, pulso com 116 batimentos por minuto, frequência respiratória com 35 incurssões respiratórias por minuto. $\mathrm{O}$ exame físico abdominal revelou distensão e dor abdominal intensa. O exame dos demais órgãos e sistemas corporais não revelaram alterações.

Os testes laboratoriais inicias evidenciaram aumento das enzimas hepáticas, dos marcadores de lesão renal e de CPK. Outros parâmetros avaliados encontravam-se dentro do limite da normalidade. A gasometria arterial admissional evidenciou presença de acidose metabólica compensada (Tabela 1).

Diante do quadro clínico, aventou-se-se a hipótese diagnóstica de insuficiência hepática aguda secundária ao uso de cocaína, de acordo com os critérios da The American Association for the Study of Liver Diseases. Além disso, apresentou insuficiência renal aguda oligúrica, de acordo 
com critérios KDIGO, secundária a grave rabdomiólise.

Foi realizada intubação orotraqueal, uma vez que paciente persistia com instabilidade hemodinâmica e com rebaixamento do nível de consciência. Droga vasoativa foi iniciada e bicarbonato de sódio, para neuroproteção. Contudo, paciente persistiu com piora clínico e laboratorial, a despeito das intervenções. Terapia dialítica foi indicada, em virtude de anúria e piora de marcadores de lesão renal.

Nos dias subsequentes ao internamento hospitalar, paciente evolui com hipoglicemia refratária (glicemia capilar de $48 \mathrm{mg} / \mathrm{dl}$ ) e piora dos parâmetros laboratoriais. Avaliação da equipe de transplante hepático foi requerida, mas após três de internamento hospitalar paciente evoluiu com óbito em virtude da gravidade do quadro clínico.

\section{Caso 3}

Paciente masculino, 38 anos, previamente hipertenso, alcoolista, tabagista (carga tabágica de 20 maços/ano), usuário de cocaína e crack há 20 anos. Foi admitido em Unidade de Terapia Intensiva dois dias após ser vítima de politrauma por agressão física e evoluir clinicamente com diminuição da diurese e mialgia difusa. Após episódio de agressão, paciente apresentou perda de consciência e broncoaspirou conteúdo lamacento.

Antes ao evento traumático fazia uso de crack e bebida alcóolica. Informou ainda fazer uso esporádico de anti-inflamatórios, devido a cefaleias constantes. Cerca de duas semanas antes do internamento apresentou quadro gripal, tendo iniciado auto-medicacação com ibuprofeno e paracetamol.

No exame admissional apresentava-se com sinais de encefalopatia e desorientação temporo-espacial, escala de coma de Glasgow de 10, ictérico, pressão arterial de 102×57 $\mathrm{mmHg}$, pulso 56 batimentos por minuto, frequência respiratória 20 incurssões respiratórias por minuto. Os demais órgãos e sistemas avaliados não apresentavam alterações.

Os testes laboratoriais inicias revelaram (Tabela 1) aumento das enzimas de lesão e função hepática, de marcadores de lesão renal e de CPK. Outros parâmetros avaliados encontravam-se dentro dos limites da normalidade. A gasometria arterial inicial evidenciou presença de acidose metabólica. Sorologias para hepatites virais e lepstospirose foram negativas, assim como as hemoculturas solicitadas.

Diante do quadro clínico, aventou-se-se a hipótese diagnóstica de Insuficiência Hepática Aguda secundária ao uso de cocaína, de acordo com os critérios da The American Association for the Study of Liver Diseases. Além disso, apresentou insuficiência renal aguda oligúrica, de acordo com critérios KDIGO, secundária a grave rabdomiólise.

Foi iniciado hidratação venosa vigorosa, rigoroso controle da função renal e indicada terapia dialítica, em virtude de anúria e azotemia. Nos dias subsequentes ao internamento paciente evolui com picos pressóricos importantes, sendo otimizada terapia anti-hipertensiva. Foi solicitada ultrassom abdominal total e com doppler de artérias renais para investigação de hipertensão secundária, os quais não evidenciaram alterações.

Paciente foi avaliado pela equipe de transplante quanto a necessidade de transplante hepático. Contudo, o mesmo não preenchia os critérios de King's College necessários.

Durante internamento hospitalar evoluiu com síndrome da abstinência e foi iniciado Lorazepam para controle dos sintomas. No curso do internamento, paciente apresentou melhora do parâmetros clínicos e laboratoriais. Após 35 dias de internamento hospitalar, paciente recebe alta para posterior seguimento ambulatorial.

\section{DISCUSSÃO}

A partir de meados do século XX ocorreu uma popularização da cocaína, com aumento significativo do uso da droga em todo mundo. Seu uso em excesso pode causar comprometimentos multisistêmicos, com alterações neurológicas, cardíacas, hepáticas, renais e psiquiátricas ${ }^{13,14}$.

No casos relatados foram observados que as anormalidades clínicas e laboratoriais no momento da admissão refletiam quadros graves, uma vez que estavam associadas a evidências de disfunções de múltiplos órgãos. A Tabela 2 descreve as características clínicas e progresso dos pacientes durante o internamento hospitalar.

O dano hepático causado pela cocaína apresenta espectro clínico amplo, variando desde alteração mínima das transaminases em usuários crônicas à hepatite aguda grave associada com rabdomiólise. O consumo de álcool em concomitância age em sinergismo para a piora da hepatotoxicidade ${ }^{15}$.

A cocaína é uma das drogas ilícitas que mais coocorrem ao abuso de álcool. Sua combinação fomenta a formação de cocaetileno, potencializando os efeitos cardiotóxicos e neurotóxicos das drogas ${ }^{16}$. Um estudo transversal com adolescentes mostrou uma associação do abuso de cocaína-crack com o álcool em $43.2 \%{ }^{17}$. O perfil dos pacientes descritos no presente estudo apresenta consonância com dados descrito na literatura, uma vez que nos três casos se observa o uso de múltiplas drogas.

O diagnóstio de hepatite aguda grave foi estabelecido a partir do quadro clínico dos pacientes no momento da admissão, com menos de 24 semanas de evolução, correlacionado aos exames laboratoriais, nos quais foram observadas alterações dos parâmetros de coagulação, elevação substâncial de transaminases, lactato desidrogenase e bilirrubinas. 
Paiva JHHGL, et al. Hepatite aguda secundária ao abuso de cocaína, associada a severa rabdomiólise.

Tabela 2. Características clínicas e progresso durante o internamento hospitalar

\begin{tabular}{lccc}
\hline Características Clínicas & Paciente $\mathbf{1}$ & Paciente 2 & Paciente 3 \\
Tempo de internamento & 29 & 3 & 35 \\
Gênero & $\mathrm{M}$ & $\mathrm{M}$ & $\mathrm{M}$ \\
\hline Idade & 32 & 33 & 38 \\
Uso de álcool em concomitância & Sim & Sim & Sim \\
\hline Uso habitual de cocaína & Sim & Sim & Sim \\
Complicações durante internamento & & & \\
Agitação psicomotora & Sim & Sim & Sim \\
Insuficiência Renal Aguda & Sim & Sim & Sim \\
\hline Encefalopatia Hepática & Sim & Sim & Sim \\
Icterícia & Sim & Sim & Sim \\
Hipotensão & Não & Sim & Não \\
Necessidade de droga vasoativa/ IOT* & Não & Sim & Não \\
Óbito & Não & Sim & Não \\
Sangramento & Sim & Não & Não \\
Pancreatite Aguda & Não & Não & Sim \\
Sorologias/Culturas & & & \\
Hepatite A & NR & $* *$ & NR \\
Hepatite B & NR & $* *$ & NR \\
Hepatite C & NR & $* *$ & NR \\
Hemocultura & NR & $* *$ & NR \\
Urinocultura & NR & $* *$ & NR \\
\hline
\end{tabular}

Legenda: *IOT: Intubação Orotraqueal; *NR: Não reagente

Fonte: elaborado pelos autores

O dano hepático nesses pacientes pode ser atribuído a múltiplos fatores. Os três pacientes era adultos jovens, na terceira década de vida, os quais faziam uso crônico de cocaína e álcool. Este fato fomenta danos adicionais a lesão hepática aguda. Vale destacar que em um dos pacientes o uso indiscriminado de anti-inflamatórios pode ter corroborado para o agravamento do quadro clínico.

Apesar do contexto epidemiológico em que se encontram os pacientes, não existem evidências que expliquem o dano hepático como sendo secundário a hepatites agudas e leptospirose, uma vez que em dois pacientes tais sorologias foram negativas. Em um dos casos não houve tempo hábil para avaliação sorológica, em virtude de o paciente ter ido a óbito três dias após a admissão.

Pesquisas descrevem que altos níveis de aspartato aminotransferase (AST) foram associados a ocorrência de insuficiência renal aguda e morte durante a hospitalização. Ocorreu também uma tendência maior a complicações associadas a doença nesses pacientes ${ }^{13}$. Resultados semelhantes puderam ser observados, uma vez que nos três casos descritos os valores de AST e Alanine aminotransferase (ALT) estiveram aumentado em mais de 20 vezes o valor da normalidade, sendo que um destes pacientes foi a óbito. Esses achados pode sugerir que o conhecimento dos níveis de AST em pacientes admitidos por intoxicação de cocaína é preditor de prognóstico.

Nos casos descritos os pacientes necessitaram de um tempo de internamento hospitalar maior do que
15 dias, refletindo maior susceptibilidade para efeitos adversos associados ao período de internamento. Além disso, apresentaram complicações nos dias subsequentes a admissão, como lesão renal aguda, necessidade de transfusões, necessidade de altas doses de drogas vasoativas.

Estudos descrevem que níveis de AST aumentado em até três vezes do seu valor normalidade foram associados a elevação de ALT e CPK. No presente estudo observa-se uma correlação direta entre o aumento das transaminases com o aumento da CPK, e quando associada ao quadro de mialgia difusa, nos leva a pensar como diagnóstico diferencial em rabdomólise ${ }^{13}$.

Rabdomiólise é uma condição séria, em que um insulto causa necrose muscular, levando à liberação sistêmica de conteúdo intracelular. Em casos graves pode desencadear lesão renal aguda devido ao efeito tóxico da mioglobina nas células renais ${ }^{4}$. A rabdomiólise pode ser estratificadas em cinco categorias etiológicas: física (lesão por esmagamento, trauma, queimaduras, convulsões), hipóxica (síndrome compartimental, imobilização, trombose vascular), química (intoxicações exógenas), biológica (acidose diabética, picadas de insetos, veneno de cobra) e genética ${ }^{18}$.

Embora não haja estudos semelhantes na literatura, o uso de cocaína tem sido relacionados à ocorrência de rabdomiólise. Danos musculares podem ser potencializados por vários fatores, como o efeito miotóxico direto da droga, vasoconstricção, isquemia, aumento da psicomotricidade 
e agitação.

Um estudo de caráter prospectivo realizado com 39 pacientes usuários crônicos de cocaína do Centro Médico Universitário de Miame-Jackson mostrou a presença de evidências consistentes com rabdomólise em 20 pacientes, que se apresentaram com mialgia difusa e aumento de CPK mais do que dez vez do limite da normalidade. Sendo que dos pacientes que desenvolveram rabdomiólise, 13 apresentaram algum grau de disfunção renal, com aumento dos valores de cretinina sérica, e 13 pacientes desenvolveram Insuficiência Hepática Aguda ${ }^{19}$. Ademais, aproximadamente $24 \%$ dos usuários de cocaína podem apresentar algum grau de rabdomiólise, sendo que o dignóstico pode ser pouco previsível pela história ou exame físico, tornando avaliação laboratorial essencial ${ }^{20}$.

Um estudo retrospectivo com 102 pacientes que estiveram sob cuidados intensivos de um centro toxicológico terciário evidenciou a prevalência de hepatite aguda grave e abuso de cocaína em 33\% dos pacientes, e $11 \%$ destes apresentaram rabdomiólise grave ${ }^{21}$.

Os três pacientes relatados desenvolveram rabdomiólise de causa multifatorial - cocaína, politrauma, agitação psicomotora - com elevação maior que 10 vezes dos valores de referência de CPK. Em todos os casos descritos, os pacientes evoluíram com Injúria Renal Aguda dialítica, predizendo uma situação clínica grave.

Na rabdomiólise o aumento de CPK atinge o pico entre 24 e 72 horas, sendo a meia-vida de aproximadamente 36 horas. Comumente, os níveis séricos voltam ao normal entre 3 a 5 dias após a injúria ${ }^{4}$. Nos casos relatados observamos que os níveis séricos de CPK permanecem elevadas por mais de dez dias, evidenciando uma maior gravidade dos pacientes em nosso serviço.

Relatos de casos sugerem uma associação entre alterações neurológicas da rabdomiólise ao uso crônico de cocaína. As alterações descritas são delírio, psicose, agitação, rebaixamento do nível de consciência ${ }^{11,22}$. Dos pacientes apresentados, um apresentou psicose e dois desorientação. A psicose secundária à rabdomiólise em usuários de cocaína, pode aumentar o risco de lesão muscular, fomentando uma piora do quadro clínico.

\section{CONCLUSÃO}

Apesar das incertezas dos mecanismos fisiopatológicos correlacionados à hepatotoxicidade secundária ao uso de cocaína, casos como os relatados têm se apresentado com maior frequência. A associação da rabdomiólise com hepatite aguda grave corrobora para uma maior gravidade com elevada morbi-mortalidade. Nesse contexto, os casos confirmam a assertiva de que todos os sistemas podem ser afetado pela toxicidade por cocaína, e sugere a importância do diagnóstico precoce e abordagem terapêutica adequada. Isto é especialmente importante porque ainda não há antídoto específico disponível para cocaína. A comunidade médica deve estar ciente das possíveis complicações do uso dessa substância, bem como da pequena diferença entre a dose recreativa e tóxica da cocaína.

Conflitos de interesses: Todos os autores declaram não haver conflitos de interesses.

Participação dos autores: Paiva JHHGL - Coleta dos dados e elaboração do artigo. Medeiros VN - Coleta dos dados e elaboração do artigo. Brasil IRC - Revisão do artigo.

\section{REFERÊNCIAS}

1. Haas C, Karila L, Lowenstein W. Cocaine and crack addiction: a growing public health problem. Bull Acad Natl Med. 2009;193:947-62.

2. Word Drug Report 2016. Cocaine. Chap. 1.Available from: http:// www.unodc.org/doc/wdr2016/WDR_2016_ Chapter_1_Cocaine.pdf.

3. Rigacci R, Madruga CS, Ribeiro M, Pinsky I, Caetano R, Laranjeira R. Addictive behaviors prevalence of cocaine use in Brazil: data from the II Brazilian National Alcohol and Drugs Survey (BNADS). Addict Behav. 2014;39(1):297-301. doi: 10.1016/j.addbeh.2013.10.019.

4. Goldberg A. Superimposed cocaine-induced rhabdomyolysis in a patient with aortic dissection rhabdomyolysis. A\&A Case Rep. 2015;4(6):75-7. doi: 10.1213/XAA.0000000000000122.

5. Ansari M, Arshed S, Islam M, Sen S, Yousif A. A case of reversible drug-induced liver failure. Clin Case Rep. 2017;5(7):1181-3. doi: 10.1002/ccr3.1030.

6. Lucey MR, Terrault N, Ojo L, Hay JE, Neuberger J, Blumberg
E, et al. Long- term management of the successful adult liver transplant: 2012 practice guideline by the American Association for the Study of Liver Diseases and the American Society of Transplantation. Liver Transpl. 2013;19:3-26. doi: 10.1002/It.

7. Hurtova M, Duclos-Vallée JC, Saliba F, Emile JF, Bemelmans $\mathrm{M}$, Castaing D, et al. Liver transplantation for fulminant hepatic failure due to cocaine intoxication in an alcoholic hepatitis C virus-infected patient. Transplantation. 2002;73(1):157-8. doi: 10.1097/00007890-200201150-00031.

8. Payancé A, Scotto B, Perarnau JM, de Muret A, Bacq Y. Severe chronic hepatitis secondary to prolonged use of ecstasy and cocaine. Clin Res Hepatol Gastroenterol. 2013;37(5):e109-13. doi: 10.1016/j.clinre.2013.06.003.

9. Roqué A, Soy G, Retto O, Núñez C, Fort E, Aldeguer X, et al. Cocaine-induced Fulminant Hepatitis. J Gastrointest Dig Syst. 2017;7(3):505. doi: 10.4172/2161-069X.1000505.

10. Tsai J-P, Lee C-J, Subeq Y-M, Lee R-P, Hsu B-G. Acute alcohol intoxication exacerbates rhabdomyolysis-induced acute renal failure in rats. Int J Med Sci. 2017;14(7):680-9. doi: $10.7150 /$ ijms. 19479 . 
Paiva JHHGL, et al. Hepatite aguda secundária ao abuso de cocaína, associada a severa rabdomiólise.

11. Kotbi N, Oliveira E, Francois D, Odom A. Mania, cocaine, and rhabdomyolysis: a case report. Am J Addict. 2012;21(6):5701. doi: 10.1111/j.1521-0391.2012.00287.x.

12. Park J-S, Seo M-S, Gil H-W, Yang J-O, Lee E-Y, Hong $\mathrm{S}$-Y. Incidence, etiology, and outcomes of rhabdomyolysis in a single tertiary referral center. J Korean Med Sci. 2013;28(17):1194-9. doi: 10.3346/jkms.2013.28.8.1194.

13. Guollo F, Narciso-Schiavon JL, Barotto AM, Zannin M, Schiavon LL. Significance of alanine aminotransferase levels in patients admitted for cocaine intoxication. J Clin Gastroenterol. 2015;49(3):250-5. doi: 10.1097/ MCG.0000000000000056.

14. Vernaglia TVC, Leite TH, Faller S, Pechansky F, Kessler FHP, Cruz MS. The female crack users: Higher rates of social vulnerability in Brazil. Health Care Women Int . 2017;38(11):1170-87. doi: 10.1080/07399332.2017.1367001.

15. Hosseinnezhad A, Vijayakrishnan R, Farmer MJS. Acute renal failure, thrombocytopenia, and elevated liver enzymes after concurrent abuse of alcohol and cocaine. Clin Pract. 2011;1(2):35. doi: 10.4081/cp.2011.e35.

16. García-Marchena N, de Guevara-Miranda DL, Pedraz M, Araos PF, Rubio G, Ruiz JJ, et al. Higher impulsivity as a distinctive trait of severe cocaine addiction among individuals treated for cocaine or alcohol use disorders. Front Psychiatry. 2018;9:1-10. doi: 10.3389/fpsyt.2018.00026.

17. Pianca TG, Rosa RL, Ceresér KMM, de Aguiar BW, de Abrahão RC, Lazzari PM, et al. Differences in biomarkers of crack-cocaine adolescent users before/after abstinence. Drug Alcohol Depend. 2017;177:207-13. doi: 10.1016/j. drugalcdep.2017.03.043.

18. Papadatos SS, Deligiannis G, Bazoukis G, Michelongona P, Spiliopoulou A, Mylonas S, et al. Nontraumatic rhabdomyolysis with short-term alcohol intoxication - a case report. Clin Case Rep. 2015;3(10):769-72. doi: 10.1002/ ccr3.326.

19. Roth D, Alarcon FJ, Fernandez JÁ, Preston RA, Bourgoignie JJ. Acute Rhabdomyolysis associated with cocaine intoxication. N Engl J Med. 1988;319(11):673-77. doi: 10.1056/NEJM198903093201011.

20. Elnenaei MO, Heneghen MA, Moniz C. Life-threatening hyperkalaemia and multisystem toxicity following first-time exposure to cocaine. Ann Clin Biochem. 2012;49(2):197-200. doi: 10.1258/acb.2011.011095.

21. OConnor AD, Padilla-Jones A, Gerkin RD, Levine M. Prevalence of rhabdomyolysis in sympathomimetic toxicity: a comparison of stimulants. J Med Toxicol. 2015;11(2):195200. doi: 10.1007/s13181-014-0451-y.

22. Sise ME, Lo GC, Goldstein RH, Allegretti AS, Masia R. Case 12-2017 - a 34-year-old man with nephropathy. N Engl J Med. 2017;376(16):1575-85. doi: 10.1056/ NEJMcpc1616395.

Recebido: 25.08.2019

Aceito: 04.03.2020 